\title{
Qualitative behavior of numerical traveling solutions for reactiondiffusion equations with memory
}

\author{
A. ARAÚJo, J. A. FERREIRA and P. OLIVEIRA* \\ Departamento de Matemática, Universidade de Coimbra, Coimbra, Portugal
}

\begin{abstract}
In this article the qualitative properties of numerical traveling wave solutions for integrodifferential equations, which generalize the well known Fisher equation are studied. The integro-differential equation is replaced by an equivalent hyperbolic equation which allows us to characterize the numerical velocity of traveling wave solutions. Numerical results are presented.
\end{abstract}

Keywords: Fisher equation; Integro-differential equation; Traveling wave solution; Numerical approximation

1991 Mathematics Subject Classifications: 65M06; 65M20; 65M15

\section{Reaction-diffusion equations with memory}

Mathematical models based on the well-known Fisher reaction-diffusion equation

$$
\frac{\partial u}{\partial t}=D \frac{\partial^{2} u}{\partial x^{2}}+f(u), \quad(x, t) \in \mathbb{R} \times \mathbb{R}^{+}
$$

have been largely used to describe physical, chemical or biological models $[3,4,6]$. For example, we can mention models of propagation of a vortex front in an unstable fluidflow, models of aggregation and deposition, ecological models or biological invasions models. In (1) $D$ is a diffusion coefficient, $f$ is a nonlinear function with $f(u)>0$ and $f(0)=f(1)=0$.

\footnotetext{
*Corresponding author. Email: poliveir@mat.uc.pt
} 


\section{A. Araúijo et al.}

However, Fisher equations present two unphysical properties which are related. Firstly, due to its parabolic character, if a sudden change occurs at a certain point it will be felt instantly everywhere, though with exponentially small amplitudes at distant points. The second unphysical property concerns the overestimation of the velocity of traveling waves. In fact for a sufficiently localized initial condition, the solution of (1) converges to a traveling wave solution in the long time limit [1,6] connecting the two steady states: $u=0$ (unstable) and $u=1$ (stable). This means that, a solution of the type $\psi(x-c t)$ - where $\psi$ is a monotonically decreasing function such that $\psi(-\infty)=1, \psi(+\infty)=0$, and $c$ is the velocity at which the wave profile $\psi$ moves - will describe the evolution of the system between the two steady states. In several models the reaction term is represented by $f(u)=U(1-u) u$ where $U$ stands for a reaction rate parameter. In this case it is well known [6] that the velocity $c_{F}$ of the traveling wave is equal to $\sqrt{4 D U}$. When the chemical rate becomes very fast, arbitrarily large unphysical velocities arise, which contradicts the simple fact that they should not exceed the propagation rate of the real transport process.

To overcome these unphysical properties the flux $q_{F}$ used in (1) and defined by Fick's law

$$
q_{F}=-D \frac{\partial u}{\partial x}
$$

can be replaced by a flux with memory defined by

$$
q_{I}=-\frac{D}{\tau} \int_{0}^{t} e^{-(t-s) / \tau} \frac{\partial u}{\partial x}(x, s) d s
$$

where $\tau$ is a relaxation parameter [3-5]. Using (2), the mathematical models based on the parabolic Fisher equation are then replaced by the integro-differential equation

$$
\frac{\partial u}{\partial t}=\frac{D}{\tau} \int_{0}^{t} e^{-(t-s) / \tau} \frac{\partial^{2} u}{\partial x^{2}}(x, s) d s+f(u), \quad(x, t) \in \mathbb{R} \times \mathbb{R}^{+}
$$

The integro-differential equation (3), which leads to the Fisher equation when $\tau$ converges to zero, is known as a generalized Fisher-Kolmogorov-Petrovski-Piskunov equation (FKPP) and is considered, for instance, in [3-5]. Integro-differential equations of type (3) have also been considered in [2,10,11] (see [9] for other Integro-differential models).

The computation of the velocity $c_{I}$ of a traveling wave solution of (3) has attracted considerable interest in the past years (see, for instance, [3-5,8]). Considering an initial condition of Heaviside type, the authors established in [3] that

$$
c_{I}=\frac{\sqrt{4 D U}}{1+\tau U}
$$

with $f(u)=U u(1-u)$, where $U$ is a constant and $\tau U \leq 1$. This result was generalized in [4] for a reaction term of type $f(u)=U(x) u(1-u)$. The overestimation of $c_{F}$ is corrected by flux (2) because $c_{I} \leq c_{F}$. We remark that considering $c_{I}$ as a function of $U$ and $\tau$, 
in the domain defined by $\tau U \leq 1$, we can easily establish that $c_{I} \leq \sqrt{D} \tau$, where this maximum is attained for $U \tau=1$.

In the present article, we are concerned with the construction of numerical methods to solve (3), which present accurate numerical velocities. One possible approach is the use of finite differences for the discretization of partial derivatives and quadrature formulas for the integral term. This method was considered, for instance, in $[2,10]$. Another approach is the use of Galerkin method (see, for instance, [11] and the references cited therein). The method proposed in this article avoids the discretization of the integral term and is based on the discretization of a partial differential equation equivalent to (3): the telegraph equation

$$
\frac{\partial^{2} u}{\partial t^{2}}+\frac{\partial u}{\partial t}\left(\frac{1}{\tau}-f^{\prime}(u)\right)=\frac{D}{\tau} \frac{\partial^{2} u}{\partial x^{2}}+\frac{1}{\tau} f(u) .
$$

In section 2 we establish, under certain conditions, the equivalence between the integro-differential equation (3) and the telegraph equation, and we study the sensivity of the models relatively to initial conditions. In section 3 we study the behavior of two simple numerical methods for solving the integro-differential equation (3) obtained by considering the discretization of the equivalent telegraph equation. The numerical speeds of the methods are studied. Numerical simulations are also included.

\section{The generalized FKPP equation versus the telegraph equation}

In this section we prove the equivalence between the integro-differential equation (3) and a partial differential equation of second order in time - the telegraph equation. We note that, to describe reaction-diffusion processes, several authors $[7,8]$ have used the telegraph equation. The sensivity of the integro-differential equation relatively to the initial conditions is also established in what follows.

If $f \in C^{1}$, we have, from (3),

$$
\frac{\partial^{2} u}{\partial t^{2}}(x, t)=\frac{D}{\tau} \frac{\partial^{2} u}{\partial x^{2}}(x, t)-\frac{D}{\tau^{2}} \int_{0}^{t} e^{-(t-s) / \tau} \frac{\partial^{2} u}{\partial x^{2}}(x, s) d s+f^{\prime}(u) \frac{\partial u}{\partial t}
$$

and consequently we obtain (5). If (3) is coupled with the initial condition

$$
u(x, 0)=u_{0}(x), \quad x \in \mathbb{R},
$$

then for the telegraph equation (5) we have the following initial conditions:

$$
\begin{cases}u(x, 0)=u_{0}(x), & x \in \mathbb{R}, \\ \frac{\partial u}{\partial t}(x, 0)=f\left(u_{0}(x)\right), & x \in \mathbb{R} .\end{cases}
$$




\section{A. Araújo et al.}

Inversely, if $u$ is a solution of the telegraph equation (5) with initial conditions

$$
\begin{cases}\frac{\partial u}{\partial t}(x, 0)=q(x), & x \in \mathbb{R} \\ u(x, 0)=p(x), & x \in \mathbb{R}\end{cases}
$$

then $u$ is a solution of the modified integral equation

$$
\frac{\partial u}{\partial t}=\frac{D}{\tau} \int_{0}^{t} e^{-(t-s) / \tau} \frac{\partial^{2} u}{\partial x^{2}}(x, s) d s+f(u)+(q(x)-f(p(x))) e^{-t / \tau} .
$$

In fact (5) is equivalent to

$$
\frac{\partial}{\partial t}\left(\frac{\partial u}{\partial t} e^{t / \tau}\right)=\frac{D}{\tau} \frac{\partial^{2} u}{\partial x^{2}} e^{t / \tau}+\frac{\partial}{\partial t}\left(f(u) e^{t / \tau}\right) .
$$

Integrating this equation we obtain

$$
\frac{\partial u}{\partial t}=\frac{D}{\tau} \int_{0}^{t} e^{-(t-s) / \tau} \frac{\partial^{2} u}{\partial x^{2}} d s+f(u)+e^{-t / \tau} g(x),
$$

where $g(x)$ stands for an integration constant. Attending to (9) we finally establish (10).

The modified integral equation (10) coincides with equation (3) if and only if $q(x)=f(p(x)), u_{0}(x)=p(x), x \in \mathbb{R}$.

We summarize the previous considerations in the following result:

Proposition 1 Let $u$ be a solution of (3) with initial condition (7) and $v$ a solution of (5) with initial conditions (9). Then $u=v$ if and only if $q(x)=f(p(x)), p(x)=u_{0}(x)$, $x \in \mathbb{R}$.

Equation (5) is an hyperbolic equation with characteristics defined by $d x / d t= \pm \sqrt{D / \tau}$. As $\tau$ increases, the "memory" of the process also increases. In fact, the angular coefficient of the characteristics is a decreasing function of $\tau$ and consequently a greater time interval, $[t-x \sqrt{\tau / D}, t+x \sqrt{\tau / D}]$, is involved in the computation of $u(x, t)$ (see figure 1$)$.

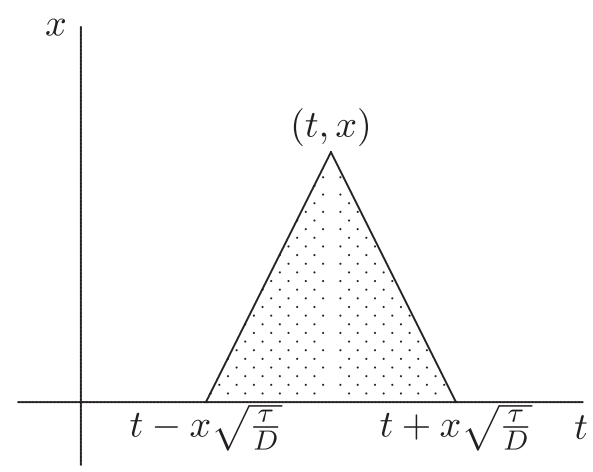

Figure 1. Domain of dependence. 
As a consequence, the first unphysical property of parabolic Fisher equation is obviously corrected by hyperbolic equation (5); a change that occurs at a certain point $x$ will not be felt instantly everywhere, but after an elapsed time of $x \sqrt{\tau / D}$. When $\tau \rightarrow 0$ the two characteristics of the hyperbolic equation degenerate into a only one vertical characteristic.

Another consequence of the hyperbolic character of the telegraph equation is that the total energy associated with (5) satisfies

$$
\frac{d}{d t}\left(\left\|\frac{\partial u}{\partial t}\right\|_{L^{2}(\mathbb{R})}^{2}+\frac{D}{\tau}\left\|\frac{\partial u}{\partial x}\right\|_{L^{2}(\mathbb{R})}^{2}-\int_{\mathbb{R}} g(u) d x\right)=2 \int_{\mathbb{R}}\left(\frac{f^{\prime}(u) \tau-1}{\tau}\left(\frac{\partial u}{\partial t}\right)^{2}\right) d \xi
$$

where $g(u)=1 / 2 \int_{0}^{u} f(\xi) d \xi$.

If $1-\tau f^{\prime}(u) \geq 0$, we obtain from (11)

$$
\left\|\frac{\partial u}{\partial t}\right\|_{L^{2}(\mathbb{R})}^{2}+\frac{D}{\tau}\left\|\frac{\partial u}{\partial x}\right\|_{L^{2}(\mathbb{R})}^{2}-\int_{\mathbb{R}} g(u) d x \leq\left\|f\left(u_{0}\right)\right\|_{L^{2}(\mathbb{R})}^{2}+\frac{D}{\tau}\left\|u_{0}^{\prime}\right\|_{L^{2}(\mathbb{R})}^{2}-\int_{\mathbb{R}} g\left(u_{0}\right) d x .
$$

We now study the sensivity of the integral equation relatively to the initial conditions.

Proposition 2 Let $u$ be a solution of (3) with initial condition (7) and $v$ a solution of (10) with initial condition $v(x, 0)=p(x)$. If $u_{0}$ and $p$ have compact support in $\mathbb{R}$, then for $w=u-v$ holds the following inequality

$$
\|w\|_{L^{2}(\mathbb{R})}^{2}+\frac{D}{\tau}\left\|\int_{0}^{t} e^{-(t-s) / \tau} \frac{\partial w}{\partial x} d s\right\|_{L^{2}(\mathbb{R})}^{2} \leq e^{M t}\left\|u_{0}-p\right\|_{L^{2}(\mathbb{R})}^{2}+\|q-f(p)\|_{L^{2}(\mathbb{R})}^{2} \tau \frac{e^{M t}-e^{-t / \tau}}{1+\tau M}
$$

where $M=\max \left\{-(2 / \tau), 2 f_{\max }^{\prime}+1\right\}$.

Proof The solution $w$ satisfies the following equation

$$
\frac{\partial w}{\partial t}=\frac{D}{\tau} \int_{0}^{t} e^{-(t-s) / \tau} \frac{\partial^{2} w}{\partial x^{2}}(x, s) d s+f(u)-f(v)-(q(x)-f(p(x))) e^{-t / \tau}
$$

Multiplying this last equation by $w$ with respect to the $L^{2}(\mathbb{R})$ inner product we obtain

$$
\begin{aligned}
\frac{1}{2} \frac{d}{d t}\|w\|_{L^{2}(\mathbb{R})}^{2}= & -\frac{D}{\tau} \int_{\mathbb{R}} \int_{0}^{t} e^{-(t-s) / \tau} \frac{\partial w}{\partial x}(x, s) \frac{\partial w}{\partial x}(x, t) d s d x \\
& +\int_{\mathbb{R}}(f(u)-f(v)) w d x+e^{-t / \tau} \int_{\mathbb{R}}(f(p)-q) w d x
\end{aligned}
$$




\section{A. Araújo et al.}

Using integration by parts it can be established that

$$
\begin{aligned}
\frac{D}{\tau} \int_{\mathbb{R}} \int_{0}^{t} e^{-(t-s) / \tau} \frac{\partial w}{\partial x}(x, s) \frac{\partial w}{\partial x}(x, t) d s d x= & \frac{d}{d t} \frac{1}{2} \frac{D}{\tau}\left\|\int_{0}^{t} e^{-(t-s) / \tau} \frac{\partial w}{\partial x} d s\right\|_{L^{2}(\mathbb{R})}^{2} \\
& +\frac{D}{\tau^{2}}\left\|\int_{0}^{t} e^{-(t-s) / \tau} \frac{\partial w}{\partial x} d s\right\|_{L^{2}(\mathbb{R})}^{2}
\end{aligned}
$$

As the inequalities

$$
\int_{\mathbb{R}}(f(u)-f(v)) w d x \leq f_{\max }^{\prime}\|w\|_{L^{2}(\mathbb{R})}^{2}
$$

and

$$
e^{-(t / \tau)} \int_{\mathbb{R}}(f(p)-q) w d x \leq \frac{1}{2} e^{-(t / \tau)}\|f(p)-q\|_{L^{2}(\mathbb{R})}^{2}+\frac{1}{2}\|w\|_{L^{2}(\mathbb{R})}^{2}
$$

hold, we establish from (14)-(17)

$$
\frac{d}{d t} E(w) \leq M E(w)+e^{-(t / \tau)}\|f(p)-q\|_{L^{2}(\mathbb{R})}^{2},
$$

with $M=\max \left\{-(2 / \tau), 2 f_{\max }^{\prime}+1\right\}$ and

$$
E(w)=\|w\|_{L^{2}(\mathbb{R})}^{2}+\frac{D}{\tau}\left\|\int_{0}^{t} e^{-(t-s) / \tau} \frac{\partial w}{\partial x} d s\right\|_{L^{2}(\mathbb{R})}^{2}
$$

Integrating the differential inequality (18) we obtain (13).

If $f(u)=0$ we conclude from (13) that $E(w) \rightarrow 0$ when $t \rightarrow \infty$. If $f(u) \neq 0$ and $f_{\max }^{\prime}>0,(13)$ just establishes that $E(w)$ is bounded for any fixed time $T$. In the context of loss reaction equations we have $f^{\prime}<0$ and consequently estimation (13) allow us to conclude that $E(w)$ is decreasing.

In the following we compare estimate (12) with estimate (13) for the particular choice $f(u)=u$. From (12) we deduce

$$
\left\|\frac{\partial u}{\partial t}\right\|_{L^{2}(\mathbb{R})}^{2}+\frac{D}{\tau}\left\|\frac{\partial u}{\partial x}\right\|_{L^{2}(\mathbb{R})}^{2}-\|u\|_{L^{2}(\mathbb{R})}^{2} \leq\left\|f\left(u_{0}\right)\right\|_{L^{2}(\mathbb{R})}^{2}+\frac{D}{\tau}\left\|u_{0}^{\prime}\right\|_{L^{2}(\mathbb{R})}^{2}-\frac{1}{\tau}\left\|u_{0}\right\|_{L^{2}(\mathbb{R})}^{2} .
$$

Otherwise from (13) we obtain

$$
\|u\|_{L^{2}(\mathbb{R})}^{2}+\frac{D}{\tau}\left\|\int_{0}^{t} e^{-(t-s) / \tau} \frac{\partial u}{\partial x} d s\right\|_{L^{2}(\mathbb{R})}^{2} \leq e^{2 t}\left\|u_{0}\right\|_{L^{2}(\mathbb{R})}^{2}
$$


Inequality (20) improves the information given by (19). In fact from (20) we obtain an upper bound to the difference between the total "energy" - kinetic and potential - $\|\partial u / \partial t\|_{L^{2}(\mathbb{R})}^{2}+D / \tau\|\partial u / \partial x\|_{L^{2}(\mathbb{R})}^{2}$ and the total concentration $\|u\|_{L^{2}(\mathbb{R})}^{2}$. But we are not able to conclude that each part of this difference is bounded. From (20) we conclude that the total concentration and the norm of the history of the gradient are bounded.

\section{Numerical methods for the generalized FKPP equation}

In this section we present simple first order numerical methods for solving the integro-differential equation (3). These methods are established from Proposition 1, that is by using finite difference discretizations of (5) with $t_{\epsilon}\left[\mathrm{O}_{1} \mathrm{~T}\right]$.

We consider a spatial uniform grid $x_{i}$ such that $x_{i+1}-x_{i}=h$ and a uniform temporal grid $t_{n}$ such that $t_{n+1}-t_{n}=k$. By $u_{j}^{n}$ we denote a numerical approximation of $u\left(x_{j}, t_{n}\right)$.

Let us consider equation (5) at $\left(x_{j}, t_{n}\right)$. We consider numerical methods obtained by discretizing $\left(\partial^{2} u / \partial t^{2}\right)\left(x_{j}, t_{n}\right)$ and $(\partial u / \partial t)\left(x_{j}, t_{n}\right)$ respectively with second order and backward finite difference operators.

\subsection{An explicit method}

3.1.1. Convergence and qualitative behavior. Let us consider the explicit method $D_{n} R_{n}$ defined by

$$
D_{2, t} u_{j}^{n}+D_{-t} u_{j}^{n}\left(\frac{1}{\tau}-f^{\prime}\left(u_{j}^{n}\right)\right)=\frac{D}{\tau} D_{2, x} u_{j}^{n}+\frac{1}{\tau} f\left(u_{j}^{n}\right)
$$

where

$$
D_{-t} u_{j}^{i}=\frac{u_{j}^{i}-u_{j}^{i-1}}{k}, \quad D_{2, x} u_{j}^{i}=\frac{u_{j+1}^{i}-2 u_{j}^{i}+u_{j-1}^{i}}{h^{2}}, \quad D_{2, t} u_{j}^{i}=\frac{u_{j}^{i+1}-2 u_{j}^{i}+u_{j}^{i-1}}{k^{2}} .
$$

To study the local stability in the neighborhood of the unstable steady state $u=0$ (where $f^{\prime}(0)>0$ ) we use a von Neumann analysis in the linearized method. For the sake of simplicity let us assume that $\tau f^{\prime}(0)=1$. It can be shown that the amplification factor $\xi$ satisfies

$$
|\xi| \leq 1+k f^{\prime}(0)
$$

provided that

$$
\frac{D k^{2}}{h^{2} \tau} \leq \frac{1}{2},
$$

and

$$
k \leq \frac{1}{f^{\prime}(0)^{2}} .
$$




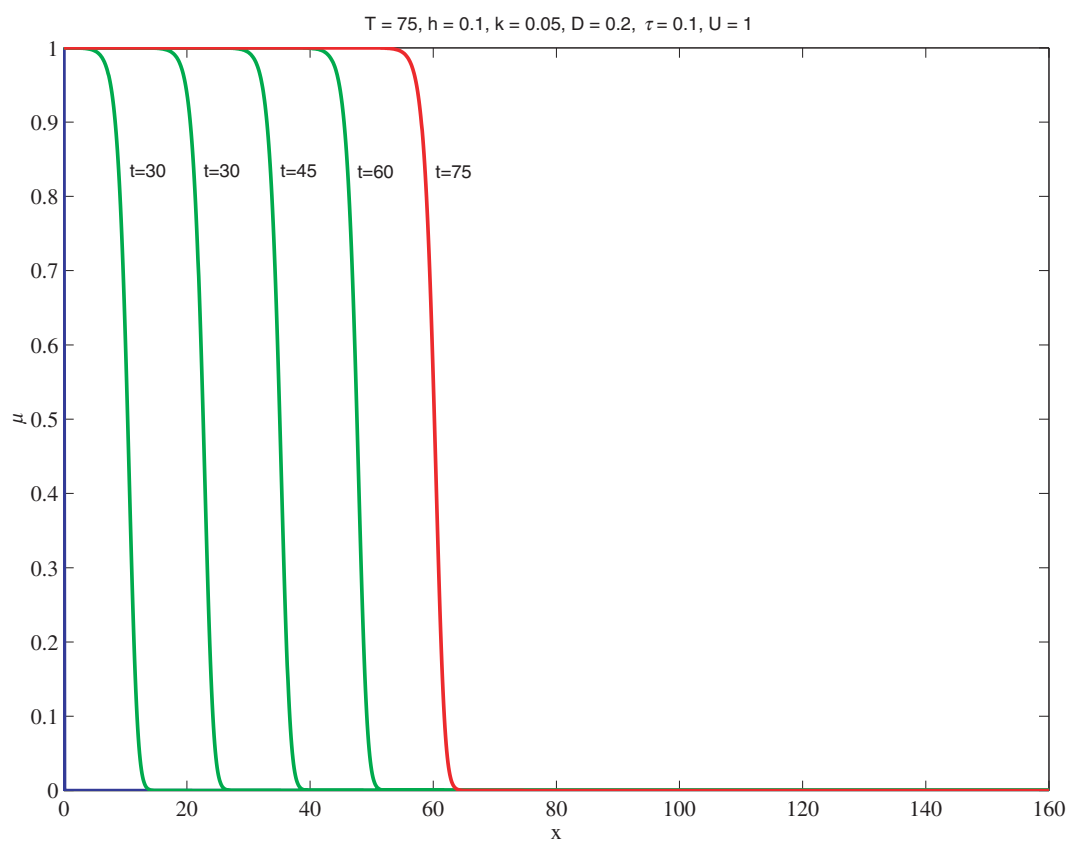

Figure 2. Numerical solutions obtained by method $D_{n} R_{n}-$ stable behaviour.

Numerical simulations obtained with method $D_{n} R_{n}$ are shown in figures 2 and 3 . The initial profile is an Heaviside function $H(x)$. The lack of stability of the method is well illustrated in figure 3 . We remark that while the data in figure 2 verify (23), the data in figure 3 violate this condition.

The diffusive behavior of method $D_{n} R_{n}$ can be understood by constructing the modified partial differential equation which exact solution is the numerical solution at the mesh nodes. If $\bar{u}$ represents the interpolation function of $u_{j}^{n}$ and assuming that this function is smooth enough then $\bar{u}$ is a solution of

$$
\frac{\partial^{2} \bar{u}}{\partial t^{2}}\left(1+\frac{k}{2} \frac{1-\tau f^{\prime}(0)}{\tau}\right)+\frac{\partial \bar{u}}{\partial t}\left(\frac{1-\tau f^{\prime}(0)}{\tau}-k \frac{f^{\prime}(0)}{\tau}\right)=\frac{D}{\tau} \frac{\partial^{2} \bar{u}}{\partial x^{2}}+\frac{f^{\prime}(0)}{\tau} \bar{u},
$$

where second-order terms have been discarded. If we consider the behavior of a plane wave of form

$$
\bar{u}(x, t)=e^{-p(m) t} e^{i m x(x-q(m) t)}
$$

when introduced in (25), we conclude that the parameter $p$, which measures diffusivity, is defined by

$$
p(m)=-\frac{1-f^{\prime}(0) \tau}{2 \tau+\left(1-f^{\prime}(0) \tau\right) k},
$$




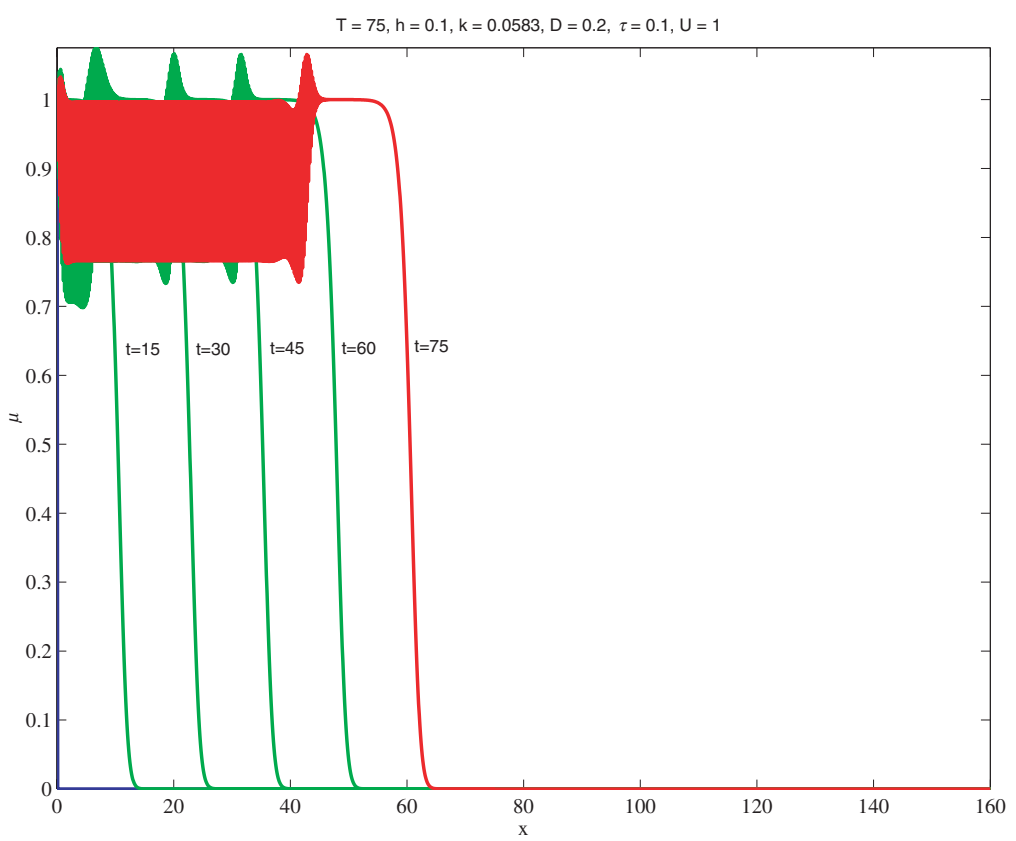

Figure 3. Numerical solutions obtained by method $D_{n} R_{n}$ - unstable behaviour.

for $m$ large enough. If the plane wave is introduced in the linearized version of (5) in the neighborhood of $u=0, p$ takes the value

$$
p(m)=-\frac{1-f^{\prime}(0) \tau}{2 \tau}
$$

which means that the exact solution of the telegraph equation presents a smaller diffusivity than the numerical solution obtained with method $D_{n} R_{n}$.

3.1.2. Velocity of propagation of traveling waves. The computation of the velocity of propagation of traveling waves is a central problem in diffusive reaction models. In this section we study the velocities of numerical wave solutions obtained from method $D_{n} R_{n}$. It is worthwhile to mention that even if we know that the solution of (3) evolves into a traveling wave of type $\psi(x-c t)$ - where $c$ is the constant velocity of propagation - the solution of the equivalent telegraph equation

$$
\psi^{\prime \prime}\left(c^{2}-\frac{D}{\tau}\right)+c \psi^{\prime}\left(\frac{1}{\tau}-f^{\prime}(\psi)\right)-\frac{1}{\tau} f(\psi)=0
$$

cannot be computed from (27) because $\psi$ and $c$ are unknowns. However, using (27), we can proceed to a stability analysis of traveling wave solutions $\psi$, near $u=0$. Solving the 
quadratic equation in $\delta$,

$$
\delta^{2}\left(c^{2} \tau-D\right)+\delta\left(-c+f^{\prime}(0) \tau c\right)-f^{\prime}(0)=0
$$

we obtain

$$
\delta=\frac{c\left(1-f^{\prime}(0) \tau\right) \pm \sqrt{\left(1-f^{\prime}(0) \tau\right)^{2} c^{2}+4\left(c^{2} \tau-D\right) f^{\prime}(0)}}{2\left(c^{2} \tau-D\right)}
$$

To obtain a real solution $\psi$, with a physical meaning, we must have the radicand positive that is

$$
c \geq \frac{\sqrt{4 f^{\prime}(0) D}}{1+\tau f^{\prime}(0)}
$$

To guarantee the stability of $\psi$ we impose $1-\tau f^{\prime}(0)>0$ and $c^{2} \tau-D<0$.

To study the numerical speed we consider a family of functions of type

$$
w_{m}(x, t)=a(m) e^{-m x+\lambda(m) t},
$$

with $m>0$, and we prove here that for a certain value of $m$ the corresponding wave propagates with a velocity defined by (4). We recall that in [3] it has been proved that a Heaviside function also propagates with the same speed.

By replacing (28) in (27) with $f(u)=f^{\prime}(0) u$ we conclude that (28) is a solution of

$$
\frac{\partial^{2} w}{\partial t^{2}}+\frac{\partial w}{\partial t}\left(\frac{1}{\tau}-f^{\prime}(0)\right)=\frac{D}{\tau} \frac{\partial^{2} w}{\partial x^{2}}+\frac{1}{\tau} f^{\prime}(0) w
$$

if and only if

$$
\lambda^{2}+\lambda\left(\frac{1}{\tau}-f^{\prime}(0)\right)-\frac{1}{\tau}\left(m^{2}+f^{\prime}(0)\right)=0
$$

The velocity $c(m), c(m)=\lambda(m) / m$, is defined by

$$
c_{+}(m)=\frac{-1+\tau f^{\prime}(0)}{2 m \tau}+\sqrt{\frac{D}{\tau}+\frac{\left(1+\tau f^{\prime}(0)\right)^{2}}{4 \tau^{2} m^{2}}}
$$

and

$$
c_{-}(m)=\frac{-1+\tau f^{\prime}(0)}{2 m \tau}-\sqrt{\frac{D}{\tau}+\frac{\left(1+\tau f^{\prime}(0)\right)^{2}}{4 \tau^{2} m^{2}}} .
$$




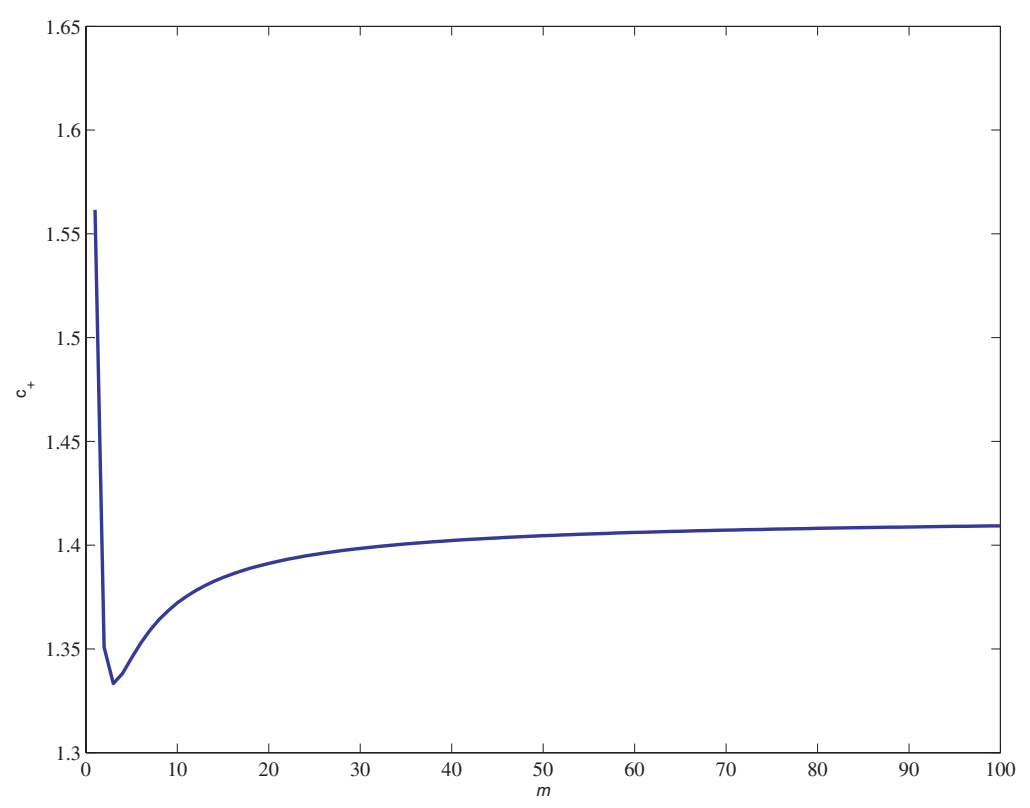

Figure 4. The graph of $c_{+}(m)$.

As we want to characterize the traveling wave solutions connecting $u=1$ with $u=0$, we consider in what follows $c_{+}(m)$. We assume that $\tau f^{\prime}(0) \leq 1$. It is a tedious but straightforward task to establish that

$$
\min _{m>0} c_{+}(m)=c_{+}\left(m^{*}\right)=\frac{\sqrt{4 D f^{\prime}(0)}}{1+\tau f^{\prime}(0)},
$$

with $m^{*}=\sqrt{\left(f^{\prime}(0) / D\right)}\left(1+\tau f^{\prime}(0)\right) /\left(1-\tau f^{\prime}(0)\right)$. In figure 4 we plot the graph of $c_{+}(m)$ for $\tau=0.5, f^{\prime}(0)=1$ and $D=1$.

As proved before, to have a stable positive traveling wave $w_{m}(x, t)$ we should have

$$
\frac{\sqrt{4 f^{\prime}(0) D}}{1+\tau f^{\prime}(0)} \leq c_{+}(m) \leq \sqrt{\frac{D}{\tau}} .
$$

As $c_{+}\left(m^{* *}\right)=\sqrt{D / \tau}$, with $m^{* *}=\sqrt{\tau / D}\left(f^{\prime}(0)\right) /\left(1-\tau f^{\prime}(0)\right)$, we conclude from (33) and (34), that $w_{m}$ is stable and positive for $m \geq m^{* *}$.

We note that traveling waves (28) with velocity $c_{+}(m)$ for $m>0$, define a family of solutions (see figure 5) of the integro-differential modified problem

$$
\left\{\begin{array}{l}
\frac{\partial u}{\partial t}=\frac{D}{\tau} \int_{0}^{t} e^{-(t-s) / \tau} \frac{\partial^{2} u}{\partial x^{2}}(x, s) d s+f^{\prime}(0) u+\left(m v_{+}(m)-f^{\prime}(0)\right) e^{-m x-(t / \tau)}, \\
(x, t) \in \mathbb{R} \times \mathbb{R}^{+} \\
u(x, 0)=e^{-m x}, \quad x \in \mathbb{R},
\end{array}\right.
$$




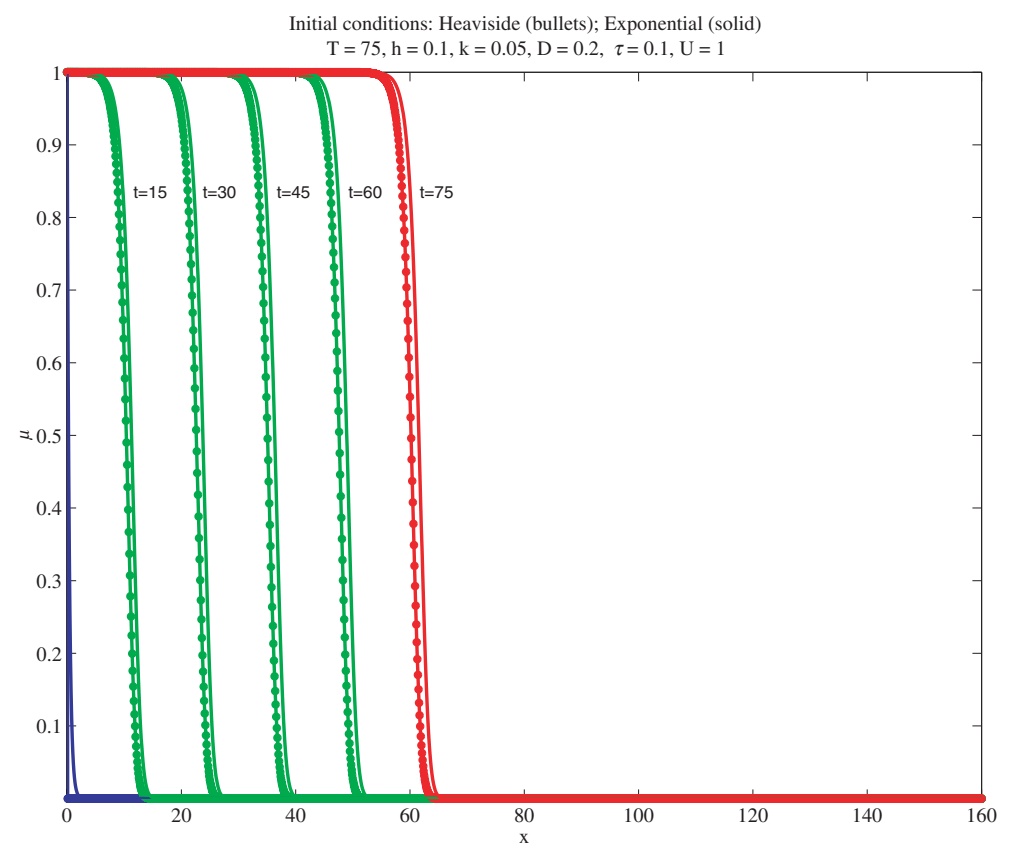

Figure 5. Traveling waves for $u_{0}(x)=H(x)$ and $u_{0}(x)=e^{m^{*}\left(-x+c_{+}\left(m^{*}\right) t\right)}$.

which is equivalent to equation (29) with initial conditions

$$
\begin{cases}\frac{\partial u}{\partial t}(x, 0)=m c_{+}(m) e^{-m x}, & x \in \mathbb{R} \\ u(x, 0)=e^{-m x}, & x \in \mathbb{R} .\end{cases}
$$

We have then constructed a family of trial functions such that, for each admissible velocity (34) there is an element of the family which propagates with this velocity.

In the following we use $c_{+}(m)$ to study the velocity of numerical traveling wave solutions computed with method $D_{n} R_{n}$.

To compute the numerical velocity we replace $u_{j}^{n}$ by a discrete traveling wave that is a discretization of (28), that is, $u_{j}^{n}=\xi^{n} e^{m j h}$, with $\xi=e^{c_{n}(m) k}$, where $c_{n}(m)$ represents the velocity of the numerical wave solution obtained by method $D_{n} R_{n}$. We have

$$
c_{n}(m)=\frac{1}{k} \ln \xi
$$

where $\xi$ is the solution of the following equation

$$
\begin{aligned}
& \xi^{2}+\xi\left(-2+k \frac{1-\tau f^{\prime}(0)}{\tau}-k^{2}\left(\frac{D}{\tau} \frac{e^{m h}+e^{-m h}-2}{h^{2}}+\frac{f^{\prime}(0)}{\tau}\right)\right) \\
& +\left(1-k \frac{1-\tau f^{\prime}(0)}{\tau}\right)=0 .
\end{aligned}
$$




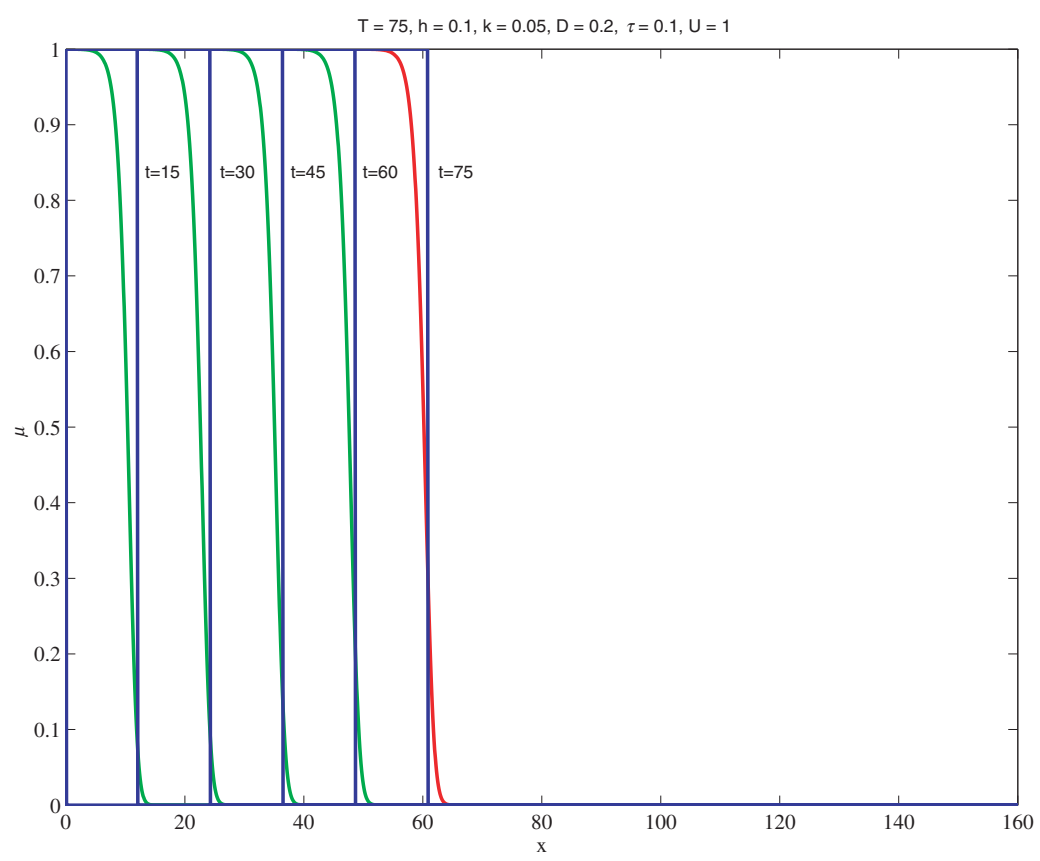

Figure 6. Numerical traveling wave solutions computed with method $D_{n} R_{n}$.

After some computations we establish that

$$
\xi=1+k\left(\frac{\tau f^{\prime}(0)-1}{2 \tau} \pm \frac{1}{2} \sqrt{\frac{\left(1+\tau f^{\prime}(0)\right)^{2}}{\tau^{2}}+4 \frac{D}{\tau} m^{2}}+O(k)\right)
$$

We have then proved the following result:

Proposition 3 Let $c_{+}(m)$ and $c_{n}(m)$ represent the speed of traveling wave solutions respectively, of the integro-differential equation (3) and of difference equation (21). Then

$$
c_{n}(m)=c_{+}(m)+O(k)
$$

In figure 6 we compare the numerical traveling wave solutions obtained with method $D_{n} R_{n}$ with the traveling wave defined by the Heaviside function and with the propagation speed $c_{+}\left(m^{*}\right)$.

\subsection{An implicit-explicit discretization}

3.2.1. Convergence and qualitative behavior. The stability of method $D_{n} R_{n}$ is achieved provided that the conditions (23) and (24) are satisfied. These conditions are very severe if the reaction is stiff. In order to avoid these stability restrictions we consider in the 


\section{A. Araújo et al.}

following an implicit-explicit method - method $D_{n+1} R_{n}$ - defined by

$$
D_{2, t} u_{j}^{n+1}+D_{-t} u_{j}^{n}\left(\frac{1}{\tau}-f^{\prime}\left(u_{j}^{n}\right)\right)=\frac{D}{\tau} D_{2, x} u_{j}^{n+1}+\frac{1}{\tau} f\left(u_{j}^{n}\right)
$$

which has an amplification factor given by

$$
|\xi| \leq 1+\frac{f^{\prime}(0)}{1-k_{0} f^{\prime}(0)} k
$$

provided that

$$
k<k_{0}<\frac{1}{f^{\prime}(0)}
$$

Numerical solutions obtained with method $D_{n+1} R_{n}$ which show better stability properties are plotted in figures 7 and 8 .

The solutions obtained with method $D_{n+1} R_{n}$ present some oscillations (figure 8). This wrong qualitative behavior can be understood by using the modified equation approach again. The modified equation associated with method $D_{n+1} R_{n}$ is defined by

$$
-\frac{D}{\tau} k \frac{\partial^{3} \bar{u}}{\partial t \partial x^{2}}+\frac{\partial^{2} \bar{u}}{\partial t^{2}}\left(1+\frac{k}{2} \frac{1-\tau f^{\prime}(0)}{\tau}\right)+\frac{\partial \bar{u}}{\partial t} \frac{1-\tau f^{\prime}(0)}{\tau}=\frac{D}{\tau} \frac{\partial^{2} \bar{u}}{\partial x^{2}}+\frac{f^{\prime}(0)}{\tau} \bar{u},
$$

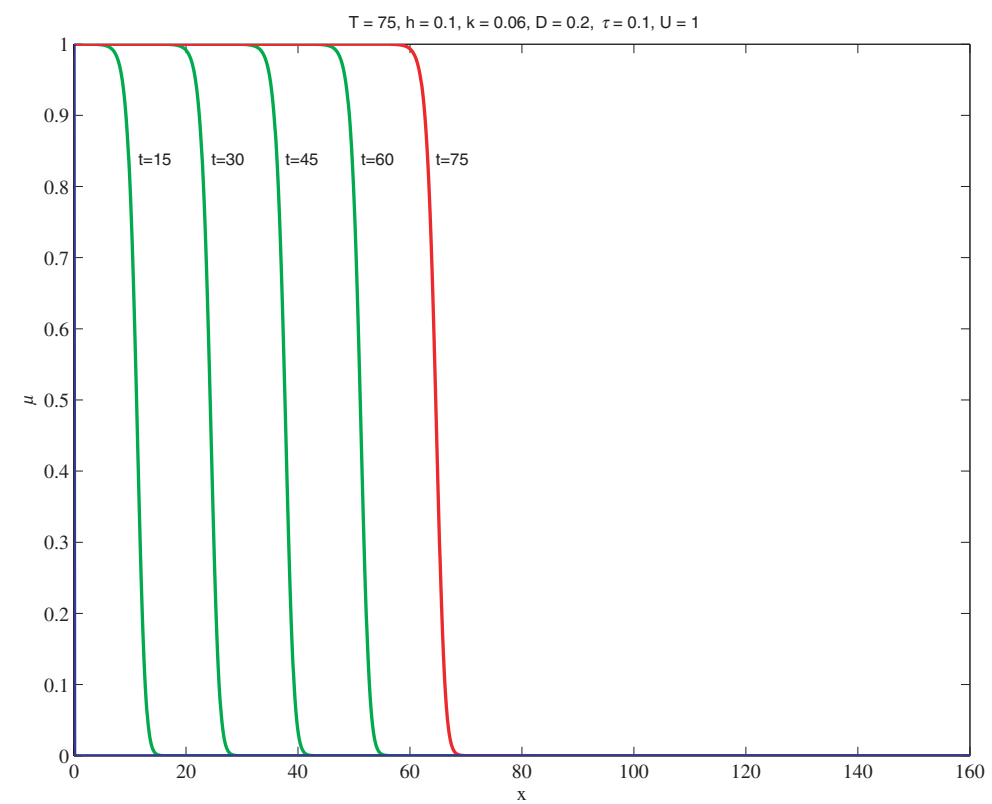

Figure 7. Numerical solution obtained using method $D_{n+1} R_{n}-$ stable behaviour. 


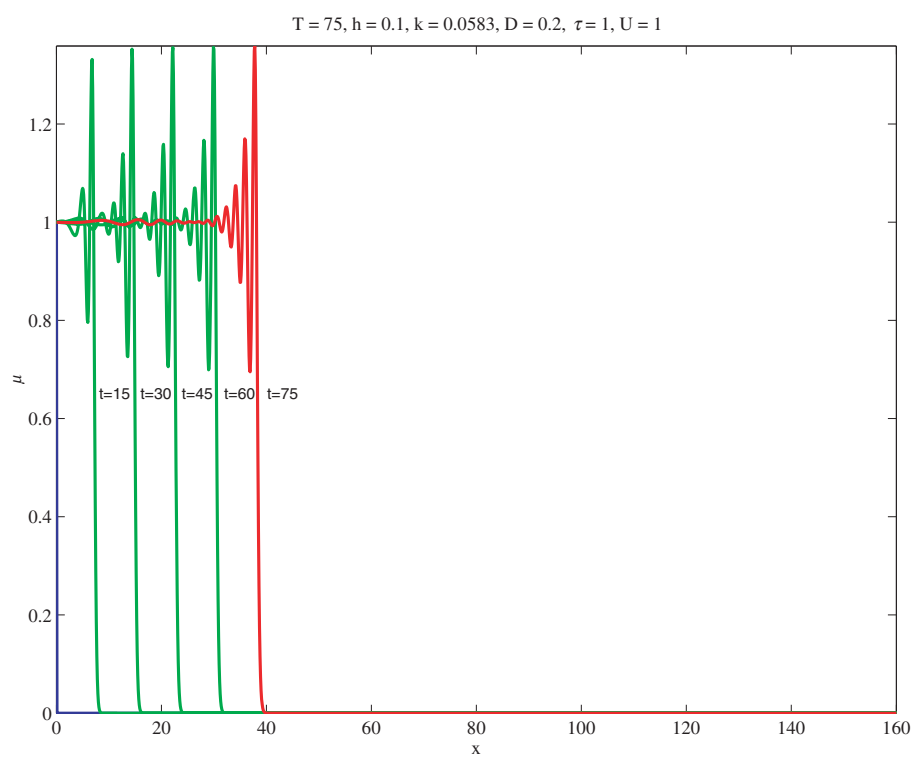

Figure 8. Numerical solutions obtained using methods $D_{n+1} R_{n}$ - oscillatory behaviour.

where second-order terms have been discarded. It is easy to show that the parameter $p$, introduced in (26), is given by

$$
p(m)=-\frac{1-f^{\prime}(0) \tau+(D k / 2) m^{2}}{2 \tau+\left(1-f^{\prime}(0) \tau\right) k}
$$

for $m$ large enough. This expression enable us to conclude that the numerical solution obtained with method $D_{n+1} R_{n}$ presents less diffusivity than the numerical solution obtained with method $D_{n} R_{n}$.

3.2.2. Velocity of traveling waves. Proceeding as previously we can conclude that Proposition 3 holds for method $D_{n+1} R_{n}$.

\section{Conclusion}

In the present article we studied the behavior of numerical traveling waves which are approximations to the solution of the integro-differential equation (3). We started by analyzing in Proposition 2 the well-posedness of the integro-differential model.

The numerical methods were constructed discretizing a partial differential equation equivalent to the integro-differential equation (3). The equivalence between the two models was established in Proposition 1.

The first method considered was of explicit type - method $D_{n} R_{n}$. This method is stable if the stepsizes satisfy conditions (23) and (24) which are very severe if stiff reactions are considered. This fact motivated the introduction of a method of implicit-explicit type - method $D_{n+1} R_{n}$. 


\section{A. Araújo et al.}

A central problem in the discrete integro-differential models is the velocity of propagation of the numerical traveling wave solutions. We established in Proposition 3 that the velocity of propagation of the numerical traveling wave solutions of method $D_{n} R_{n}$ is a first-order approximation of its continuous counterpart. The same result holds for method $D_{n+1} R_{n}$.

\section{Acknowledgment}

This work has been supported by Centro de Matemática da Universidade de Coimbra and POCTI/35039/MAT/2000.

\section{References}

[1] Aronson, D.G. and Weinberger, H.F., 1978, Multidimensional nonlinear diffusion in population genetics. Advances in Mathematics, 30, 33-76.

[2] Douglas, J. and Jones Jr., B.F., 1962, Numerical methods for integro-differential equations of parabolic and hyperbolic type. Numerische Mathematik, 4, 96-102.

[3] Fedotov, S., 1998, Traveling waves in a reaction-diffusion system: diffusion with finite velocity and Kolmogorov-Petrovski-Piskunov kinectics. Physical Review E, 5(4), 5143-5145.

[4] Fedotov, S., 1999, Nonuniform reaction rate distribution for the generalized Fisher equation: ignition ahead of the reaction front. Physical Review E, 60(4), 4958-4961.

[5] Fedotov, S., 2001, Front propagation into an unstable state of reaction - transport systems. Physical Review Letter, 86(5), 926-929.

[6] Murray, J.D., 2003, Mathematical Biology-I, II: Spatial Models and Biomedical Applications, 3rd Edn (Berlin: Springer-Verlag).

[7] Mendez, V. and Camacho, J., 1997, Dynamic and thermodynamics of delayed population growth. Physical Review E, 55(6), 6476-6482.

[8] Mendez, V. and Llebot, J., 1997, Hyperbolic reaction-diffusion equations for a forest fire model. Physical Review E, 56(6), 6557-6563.

[9] Mendez, V., Pujol, T. and Fort, J., 2002, Dispersal probability distributions and the wave front speed problem. Physical Review E, 65, 041109, 1-6.

[10] Sloan, I.H. and Thomée, V., 1986, Time discretization of an integro-differential equation of parabolic type. SIAM Journal on Numerical Analysis, 23, 1052-1061.

[11] Zhang, N.-Y., 1993, On fully discrete Galerkin approximation for partial integro-differential equations of parabolic type. Mathematics of Computation, 60(201), 133-166. 